\title{
Are atrial human pluripotent stem cell-derived cardiomyocytes ready to identify drugs that beat atrial fibrillation?
}

\author{
Torsten Christ (10) ${ }^{1,2 凶}$, Marc D. Lemoine (iD) ${ }^{2,3}$ \& Thomas Eschenhagen (1D) 1,2 \\ ARISING FROM Goldfracht et al. Nature Communications https://doi.org/10.1038/s41467-019-13868-x (2020)
}

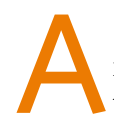

key issue in the development of atrial-selective antiarrhythmic drugs is the limited access to human heart tissue. Goldfracht et al. have used atrial- and ventricular-differentiated human embryonic stem cell-derived cardiomyocytes (hESC-CMs) to dissect chamber-selective actions of clinically relevant antiarrhythmic drugs ${ }^{1}$. This is plausible, but we want to point to remaining differences between the electrophysiological properties the atrial hESCCMs presented in the study and adult human atrial cardiomyocytes. We believe that further refinement and in-depth comparison of atrial- and ventricular-differentiated hESC-CM with adult human cardiomyocytes and tissue is warranted before these models can be safely used for the development of atrial-selective antiarrhythmics.

There is an unmet need to find new drugs to stop atrial fibrillation (AF) without inducing ventricular arrhythmias. However, access to atrial and even more to ventricular human tissue is limited, both of which could be principally solved by the widespread availability of human induced pluripotent stem cellderived cardiomyocytes (hiPSC-CM) or hESC-CMs, which are limited to some countries due to ethical requirements. Indeed, protocols to generate atrial hiPSC-CM or hESC-CM have been established ${ }^{2,3}$. However, differences in antiarrhythmic drug response between atrial and ventricular EHTs have only scarcely been investigated ${ }^{3,4}$. Therefore, the study by Goldfracht et al. using established antiarrhythmic drugs is very welcomed ${ }^{1}$. The authors used the early method of ring-shaped $\mathrm{EHTs}^{5}$, which allowed the induction of macro reentry arrhythmia, visualized by voltage sensitive dyes, and the exploration of activation maps and drug effects.

Goldfracht et al. used vernakalant, a compound approved in the EU since 2010 as an antiarrhythmic drug in AF. The authors used this multichannel blocker because it blocks, amongst others, two ion currents selectively present in the atria and not in the ventricle: the ultrarapidly activating potassium outward rectifier current $\left(I_{\text {Kur }}\right)$ and the acetylcholine-activated potassium inward rectifier current $\left(I_{\mathrm{K}, \mathrm{ACh}}\right)^{6}$. The underlying hypothesis was that effects of vernakalant in atrial, but not ventricular EHTs are indicative of a truly atrial phenotype. Indeed, vernakalant (30 $\mu \mathrm{M})$ increased action potential duration at $90 \%$ percent of repolarization $\left(\mathrm{APD}_{90}\right)$ in atrial EHTs by about $100 \%(\sim 200 \mathrm{~ms})$, while it was reported ineffective in ventricular EHTs.

Of note, however, the large effect of vernakalant on $\mathrm{APD}_{90}$ in atrial EHTs is in stark contrast to results obtained in human atrial tissue, where the same concentration of vernakalant did not prolong $\mathrm{APD}_{90}$ at all ${ }^{6}$. Inefficacy of $I_{\text {Kur }}$ block to prolong $\mathrm{APD}_{90}$ in human atrium is a common finding and is explained by indirect activation of the rapid component of the delayed rectifier potassium current $\left(I_{\mathrm{Kr}}\right)^{7}$. Thus, one has to consider block of another potassium channel to underlie the marked prolongation of $\mathrm{APD}_{90}$ in atrial EHT upon vernakalant. Contribution of $I_{\mathrm{K}, \mathrm{ACh}}$ to repolarization normally requires $G$ protein-coupled inwardly rectifying potassium channels to be open, e.g., by stimulation of muscarinic or adenosine receptors. However, agonists should be absent under in vitro conditions. Thus, other scenarios have to be considered.

Atrial hiPSC-CMs or hESC-CMs could differ from human atrium with respect to repolarization reserve (similar to what we have reported for ventricular $\mathrm{EHTs}^{8}$ ), enabling block of $I_{\text {Kur }}$ to prolong $\mathrm{APD}_{90}$.

A high baseline activity of $I_{\mathrm{K}, \mathrm{ACh}}$ could result either from acetylcholine or adenosine present in these cultures or from constitutively active $I_{\mathrm{K}, \mathrm{ACh}}$, as reported in human $\mathrm{AF}^{9}$.

Alternatively, vernakalant could have induced $\mathrm{APD}_{90}$ prolongation in atrial hESC-CM by blocking ion channels normally absent from adult CM, but present in immature hESC/hiPSC$\mathrm{CM}$, an example for this phenomenon was reported recently ${ }^{10}$.

In contrast to the unusual effect in atrial EHTs, the reported absence of effects of vernakalant on repolarization in ventricular EHTs appears, at first sight, to match the reported vernakalant

\footnotetext{
${ }^{1}$ Institute of Experimental Pharmacology and Toxicology, Cardiovascular Research Center, University Medical Center Hamburg-Eppendorf, Hamburg, Germany. ${ }^{2}$ DZHK (German Centre for Cardiovascular Research), partner site Hamburg/Kiel/Lübeck, Hamburg, Germany. ${ }^{3}$ Department of

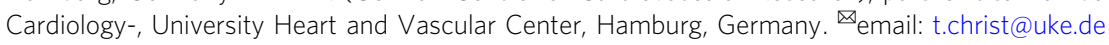


effects on human ventricular tissue ${ }^{6}$. However, the statistical approach used by Goldfracht et al. ${ }^{1}$ may be suboptimal to safely exclude effects of vernakalant. Given the clinical relevance of prolongation of repolarization, the United States Food and Drug Administration (FDA) requires the analysis of drug effects on ventricular repolarization to be done in a pairwise manner, i.e., comparing single values in the presence and absence of the test compound to detect even small changes of repolarization ${ }^{11}$. The data presented in Figure $3 \mathrm{e}$ of Goldfracht et al. ${ }^{1}$ (which have been evaluated in an unpaired manner) in fact indicate that $\mathrm{APD}_{90}$ in the vernakalant-treated group of cells was $\sim 40 \mathrm{~ms}$ longer than in the vehicle group. A randomized phase 3 clinical trials showed that vernakalant prolongs $\mathrm{QT}_{\mathrm{c}}$ in patients by $\sim 20 \mathrm{~ms}^{12}$. The effect size was confirmed in several other trials. The prolongation may be small, but could be relevant, and underlie the ongoing concern about the safety of vernakalant. In fact, FDA again rejected to approve vernakalant in the US ${ }^{13}$. We are afraid that the methodology used by Goldfracht has weakened the power to confirm established repolarization delay by vernakalant in ventricular EHT.

The authors also measured slowing of conduction as a surrogate parameter of sodium channel block. Of note, the basal upstroke velocities of ventricular and atrial EHTs action potentials in this study (12 and $7 \mathrm{~V} / \mathrm{s}$, Fig 1e) were very low in comparison to the literature $(207 \text { and } 98 \mathrm{~V} / \mathrm{s})^{3}$, and this abnormality is paralleled by depolarized take-off potentials of $>-60 \mathrm{mV}$ (Fig 1d) (normal -70 and $-76 \mathrm{mV})^{3}$. This range of upstroke velocities observed is typical of $\mathrm{Ca}^{2+}$-dependent action potentials in which $\mathrm{Ca}^{2+}$ channel provide most of the depolarizing current during the upstroke. This indicates immaturity of the hESC-CM and limits the transferability of the in vitro results with a sodium current blocker. The sodium channel blocker lidocaine $(100 \mu \mathrm{M})$ slowed down conduction in ventricular but not in atrial $\mathrm{EHT}^{1}$. The authors correctly point out that lidocaine is unable to convert AF in patients. However, we do not agree with the conclusion that the absence of lidocaine-induced conduction slowing in atrial EHT can be taken as an indication of an atrial phenotype. Even moderate concentrations of lidocaine $(\leq 10 \mu \mathrm{M})$ slowed conduction both in canine ventricle and atria ${ }^{14}$. The same concentration of lidocaine used by Goldfracht et al. $(100 \mu \mathrm{M})$ decreased both maximum upstroke velocity of action potentials and conduction velocity in atrial tissue from rabbits and guinea-pigs ${ }^{15}$. No in vitro data on human tissue are available, but the published data clearly argue against the usefulness of lidocaine to characterize an atrial drug response pattern.

Taken together, we would like to raise a word of caution when using atrial hiPSC-CMs or hESC-CMs as a model to dissect human atrial and ventricular electrophysiology. It remains unclear whether the unexpected large prolongation of $\mathrm{APD}_{90}$ by vernakalant as well as the lack of lidocaine to slow conduction is a peculiarity of this individual cell line, the culture conditions or the retinoic acid-based protocol to induce atrial differentiation. Undisputedly, the depolarized MDP and low upstroke velocity in both atrial and in ventricular cells indicate an immature, embryonic like CMs phenotype with its specific physiology and pharmacology. As a result of immaturity, disease modeling using hiPSC-CMs or hESC-CMs has been considered "of limited value if the cells are not adequately characterized with respect to their electrophysiology, contractility, kinetics, etc"16. We agree with Goldfracht et al. regarding the huge potential of atrial hiPSC-CMs or hESC-CMs. However, further refinement of atrial differentiation appears warranted to develop the full potential of these new models for cardiac electrophysiology and drug development.
Received: 24 April 2020; Accepted: 4 February 2021; Published online: 19 March 2021

\section{References}

1. Goldfracht, I. et al. Generating ring-shaped engineered heart tissues from ventricular and atrial human pluripotent stem cell-derived cardiomyocytes. Nat. Commun. 11, 1-15 (2020).

2. Lee, J. H., Protze, S. I., Laksman, Z., Backx, P. H. \& Keller, G. M. Human pluripotent stem cell-derived atrial and ventricular cardiomyocytes develop from distinct mesoderm populations. Cell Stem Cell 21, 179-194.e4 (2017).

3. Devalla, H. D. et al. Atrial-like cardiomyocytes from human pluripotent stem cells are a robust preclinical model for assessing atrial-selective pharmacology. EMBO Mol. Med. 7, 394-410 (2015).

4. Lemme, M. et al. Atrial-like engineered heart tissue: an in vitro model of the human atrium. Stem Cell Rep. 11, 1378-1390 (2018).

5. Zimmermann, W. H. et al. Tissue engineering of a differentiated cardiac muscle construct. Circ. Res. 90, 223-230 (2002).

6. Wettwer, E. et al. The new antiarrhythmic drug vernakalant: ex vivo study of human atrial tissue from sinus rhythm and chronic atrial fibrillation. Cardiovasc. Res. 98, 145-154 (2013).

7. Wettwer, E. et al. Role of IKur in controlling action potential shape and contractility in the human atrium: Influence of chronic atrial fibrillation. Circulation 110, 2299-2306 (2004).

8. Lemoine M. D., et al. Human induced pluripotent stem cell-derived engineered heart tissue as a sensitive test system for qt prolongation and arrhythmic triggers. Circ. Arrhythmia Electrophysiol. https://doi.org/10.1161/ CIRCEP.117.006035 (2018).

9. Dobrev, D. et al. The G protein-gated potassium current IK,ACh is constitutively active in patients with chronic atrial fibrillation. Circulation 112, 3697-3706 (2005).

10. Horváth, A. et al. Case report on: very early afterdepolarizations in HiPSCcardiomyocytes - an artifact by big conductance calcium activated potassium current (Ibk,Ca). Cells 9, 253 (2020).

11. FDA. Guidance for Industry Interval Prolongation and Guidance for Industry. E14 Clinical Evaluation of QT/QTc Interval Prolongation and Proarrythmic Potential for Non-Antiarrhythmic Drugs. http://www.fda.gov/cder/guidance/ index.htm; http://www.fda.gov/cber/guidelines.htm (2005). Accessed February $10,2020$.

12. Roy, D. et al. Vernakalant hydrochloride for rapid conversion of atrial fibrillation: a phase 3, randomized, placebo-controlled trial. Circulation 117, 1518-1525 (2008).

13. Dunnmon P. M., Woronow D. Vernakalant NDA 22034 Resubmission "FDA Overview of Cardiovascular Safety" "Safety of Ibutilide or Electrical Cardioversion in Patients with Atrial Fibrillation or Flutter". www.fda.gov. Accessed February 21, 2020.

14. Burashnikov, A., Di Diego, J. M., Zygmunt, A. C., Belardinelli, L. \& Antzelevitch, C. Atrium-selective sodium channel block as a strategy for suppression of atrial fibrillation: Differences in sodium channel inactivation between atria and ventricles and the role of ranolazine. Circulation 116, 1449-1457 (2007).

15. Goineau, S., Castagné, V., Guillaume, P. \& Froget, G. The comparative sensitivity of three in vitro safety pharmacology models for the detection of lidocaine-induced cardiac effects. J. Pharm. Toxicol. Methods 66, 52-58 (2012)

16. National Academies of Sciences, Engineering and M. Necessity, Use, and Care of Laboratory Dogs at the U.S. Department of Veterans Affairs. (The National Academies Press, Washington, DC, 2020). https://doi.org/10.17226/25772.

\section{Author contributions}

T.C., M.D.L., and T.E. wrote the paper.

\section{Competing interests}

The authors declare no competing interests.

\section{Additional information}

Correspondence and requests for materials should be addressed to T.C.

Peer review information Nature Communications thanks Igor Efimov, Yuji Shiba and the other, anonymous, reviewer(s) for their contribution to the peer review of this work.

Reprints and permission information is available at http://www.nature.com/reprints

\section{Data availability}

No data sets were generated or analyzed during the current study.
Publisher's note Springer Nature remains neutral with regard to jurisdictional claims in published maps and institutional affiliations. 
(c) (i) Open Access This article is licensed under a Creative Commons Attribution 4.0 International License, which permits use, sharing, adaptation, distribution and reproduction in any medium or format, as long as you give appropriate credit to the original author(s) and the source, provide a link to the Creative Commons license, and indicate if changes were made. The images or other third party material in this article are included in the article's Creative Commons license, unless indicated otherwise in a credit line to the material. If material is not included in the article's Creative Commons license and your intended use is not permitted by statutory regulation or exceeds the permitted use, you will need to obtain permission directly from the copyright holder. To view a copy of this license, visit http://creativecommons.org/ licenses/by/4.0/.

(C) The Author(s) 2021 